Aim: To gain an in-depth understanding of the lives of parents and the impact of their caregiver role on their family when they care for their child at home.

Method: An interpretative phenomenological design was deployed to interpret the narratives of sixteen parents through interviews and digital audio diaries.

Discussion: The complex journey parents undertake as caregivers to their child at home.

Conclusion: The understanding revealed will prompt practitioners to reflect on how to promote best practice for this group of children, their parents and their families by recognising the complexity of the care parents provide for their child and the support required in order to sustain their caregiver role at home.

\title{
OC13 - Parents' support needs during and following the death of their baby
}

Michaela Barnard (United Kingdom) ${ }^{1}$

\section{${ }^{1}$ University of Salford}

Theme: Complex health care and chronic disease management.

Keywords: End of life, grief, neonatal, parents, support.

Introduction: Support for bereaved parents after the death of a baby in the neonatal period is variable in the UK.

Aim: To explore parents' experiences and perceptions of support needs during and following the death of their baby.

Methods: An interpretative phenomenological study using unstructured interviews was undertaken. Analysis was undertaken using interpretative phenomenological analysis.

Results: Parental grief journeys are complicated by events experienced at the end of their baby's life. A variety of support systems are needed to facilitate parental grief journeys.

Discussion: The variation in availability of support for parents after a neonatal death needs addressing. Whilst parental need is individualised, most parents need support in some format.

Conclusions: Health professionals have a key role to play in supporting parents and signposting other supportive mechanisms after a neonatal death.

\section{OC14 - Implementation of an educational program in children's post - operative pain}

Fotini Mavridi (Greece) ${ }^{1}$; Anastasia Statiri (Greece) ${ }^{2}$;

Adelais Tsiotou (Greece)³; Evangelia Kaliardou (Greece); Anastasia Doxara (Greece) ${ }^{1}$; Vasiliki Matziou (Greece)²

${ }^{1}$ Surgical Ward, 'P\&A Kyriakou' Children's Hospital, Athens; ${ }^{2}$ National \& Kapodistrian University of Athens, Nursing Department, Athens; ${ }^{3}$ Department of Anaesthesiology, 'P\&A Kyriakou' Children's Hospital, Athens 
Theme: Nursing education, management and leadership.

Keywords: Educational intervention, paediatric pain, post-operative pain management.

Introduction: Successful pain management contributes to high-quality postoperative care.

Objective: To estimate the intensity of postoperative pain in children after an educational intervention.

Method: Surgical ward nurses after educational intervention recorded 146 children (0-16 years) postoperative pain scores, applying FLACC, FACES, NRS according to child's age during December 2012 to March 2014.

Results: The mean values of children's pain scores at the reception, $24 \mathrm{~h}$ and $48 \mathrm{~h}$ after surgery was $0,99 \pm 1,68,0,6 \pm 1,43$ and $0,13 \pm 0,79$ respectively. The median pain scores for the 1st and 2nd 24-hour seemed to correlate with the duration of surgery ( $p=0.048)$. Only $20 \%$ of cases applied some form of non-pharmacological pain method that was associated with the child's age $(p=0,001)$.

Discussion: Special care should be taken about using non-pharmacological pain control methods.

Conclusions: Postoperative pain management programs contribute to effective deal.

\title{
OC15 - Leading nursing educational progress in child mental health
}

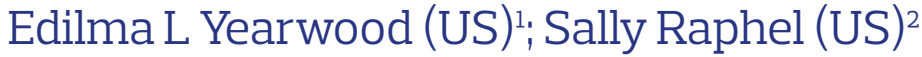 \\ ${ }^{1}$ Georgetown University School of Nursing \& Health Studies, US; ${ }^{2}$ Johns Hopkins \\ University School of Nursing, US
}

Theme: Nursing education, management and leadership.

Keywords: Child mental health, education, nurses.

Given the increasingly complex mental health needs of children and young people and the critical shortage of proficient mental health specialists, a study of US nursing education programs was done. Does the current paediatric nursing workforce preparation best meet the distinct needs of young people with behavioral and mental health challenges is the key question? Experts in the field of child psychiatric nursing practice, education and research came together to find model nursing programs for educational and clinical preparation. This presentation will report an analysis of the content of curricula-preparing psychiatric mental health nurse practitioners, models for adaptation to paediatric nurses' training and recommendations for leadership strategies.

Objectives: Describe existing US nursing education models for child and youth mental health care. Propose strategies for education leadership. 